\title{
The feasibility of predicting ground reaction forces during running from a trunk accelerometry driven mass-spring- damper model
}

\author{
Niels J Nedergaard ${ }^{1,2}$ ， Jasper Verheul ${ }^{1}$ ， Barry Drust ${ }^{1}$ ， Terence Etchells ${ }^{3}$ ， Paulo Lisboa ${ }^{3}$, Mark A Robinson ${ }^{1}$ \\ , Jos Vanrenterghem ${ }^{\text {Corresp. } 1,2}$ \\ ${ }^{1}$ Research Institute for Sport and Exercise Sciences, Liverpool John Moores University, Liverpool, United Kingdom \\ 2 Department of Rehabilitation Sciences, Katholieke Universiteit Leuven, Leuven, Belgium \\ 3 School of Computing \& Mathematical Sciences, Liverpool John Moores University, Liverpool, United Kingdom \\ Corresponding Author: Jos Vanrenterghem \\ Email address: jos.vanrenterghem@kuleuven.be
}

Background. Monitoring the external ground reaction forces (GRF) acting on the human body during running could help to understand how external loads influence tissue adaptation over time. Although mass-spring-damper (MSD) models have the potential to simulate the complex multi-segmental mechanics of the human body and predict GRF, these models currently require input from measured GRF limiting their application in field settings. Based on the hypothesis that the acceleration of the MSDmodel's upper mass primarily represents the acceleration of the trunk segment, this paper explored the feasibility of using measured trunk accelerometry to estimate the MSD-model parameters required to predict resultant GRF during running.

Methods. Twenty male athletes ran at approach speeds between $2-5 \mathrm{~m} \cdot \mathrm{s}^{-1}$. Resultant trunk accelerometry was used as a surrogate of the MSD-model upper mass acceleration to estimate the MSDmodel parameters $\left(\mathrm{ACC}_{\text {param }}\right.$ ) required to predict resultant GRF. A purpose-built gradient descent optimisation routine was used where the MSD-model upper mass acceleration was fitted to the measured trunk accelerometer signal. Root mean squared errors (RMSE) were calculated to evaluate the accuracy of the trunk accelerometry fitting and GRF predictions. In addition, MSD-model parameters were estimated from fitting measured resultant $G R F\left(G R F_{\text {param }}\right)$, to explore the difference between $A_{C C} C_{\text {param }}$ and $\mathrm{GRF}_{\text {param }}$.

Results. Despite a good match between the measured trunk accelerometry and the MSD-model's upper mass acceleration (median RMSE between 0.16 and $0.22 \mathrm{~g}$ ), poor GRF predictions (median RMSE between 6.68 and $12.77 \mathrm{~N} \cdot \mathrm{kg}^{-1}$ ) were observed. In contrast, the MSD-model was able to replicate the measured GRF with high accuracy (median RMSE between 0.45 and $0.59 \mathrm{~N} \cdot \mathrm{kg}^{-1}$ ) across running speeds from $\mathrm{GRF}_{\text {param. }}$. The $\mathrm{ACC}_{\text {param }}$ from measured trunk accelerometry under- or overestimated the $\mathrm{GRF}_{\text {param }}$ obtained from measured GRF, and generally demonstrated larger within parameter variations.

Discussion. Despite the potential of obtaining a close fit between the MSD-model's upper mass acceleration and the measured trunk accelerometry, the $\mathrm{ACC}_{\text {param }}$ estimated from this process were inadequate to predict resultant GRF waveforms during slow to moderate speed running. We therefore conclude that trunk-mounted accelerometry alone is inappropriate as input for the MSD-model to predict meaningful GRF waveforms. Further investigations are needed to continue to explore the feasibility of using body-worn micro sensor technology to drive simple human body models that would allow practitioners and researchers to estimate and monitor GRF waveforms in field settings. 


\section{The feasibility of predicting ground reaction forces during \\ 2 running from a trunk accelerometry driven mass-spring- \\ 3 damper model}

4

5 Niels J Nedergaard ${ }^{1,2}$, Jasper Verheul ${ }^{1}$, Barry Drust ${ }^{1}$, Terence Etchells ${ }^{3}$, Paulo Lisboa ${ }^{3}$, Mark A Robinson ${ }^{1}$,

6 Jos Vanrenterghem ${ }^{1,2}$

$7 \quad{ }^{1}$ Research Institute for Sport and Exercise Sciences, Liverpool John Moores University, Liverpool, United

8 Kingdom

92 Department of Rehabilitation Sciences, Katholieke Universiteit Leuven, Leuven, Belgium

${ }^{3}$ School of Computing \& Mathematical Sciences, Liverpool John Moores University, Liverpool, United

11 Kingdom

13 Corresponding Author:

14 Jos Vanrenterghem ${ }^{1,2}$

15 Tervuursevest 101 - box 1501, Leuven, 3001, Belgium

16 Email address: jos.vanrenterghem@kuleuven.be 
Abstract

19

Background. Monitoring the external ground reaction forces (GRF) acting on the human body during running could help to understand how external loads influence tissue adaptation over time. Although mass-spring-damper (MSD) models have the potential to simulate the complex multi-segmental mechanics of the human body and predict GRF, these models currently require input from measured GRF limiting their application in field settings. Based on the hypothesis that the acceleration of the MSD-model's upper mass primarily represents the acceleration of the trunk segment, this paper explored the feasibility of using measured trunk accelerometry to estimate the MSD-model parameters required to predict resultant GRF during running.

Methods. Twenty male athletes ran at approach speeds between $2-5 \mathrm{~m} \cdot \mathrm{s}^{-1}$. Resultant trunk accelerometry was used as a surrogate of the MSD-model upper mass acceleration to estimate the MSD-model parameters $\left(\mathrm{ACC}_{\text {param }}\right)$ required to predict resultant GRF. A purpose-built gradient descent optimisation routine was used where the MSD-model upper mass acceleration was fitted to the measured trunk accelerometer signal. Root mean squared errors (RMSE) were calculated to evaluate the accuracy of the trunk accelerometry fitting and GRF predictions. In addition, MSD-model parameters were estimated from fitting measured resultant GRF $\left(G R F_{\text {param }}\right)$, to explore the difference between $A C C_{\text {param }}$ and $\mathrm{GRF}_{\text {param }}$.

Results. Despite a good match between the measured trunk accelerometry and the MSDmodel's upper mass acceleration (median RMSE between 0.16 and $0.22 \mathrm{~g}$ ), poor GRF predictions (median RMSE between 6.68 and $12.77 \mathrm{~N} \cdot \mathrm{kg}^{-1}$ ) were observed. In contrast, the MSD-model was able to replicate the measured GRF with high accuracy (median RMSE between 0.45 and $0.59 \mathrm{~N} \cdot \mathrm{kg}^{-1}$ ) across running speeds from $\mathrm{GRF}_{\text {param }}$. The $A C C_{\text {param }}$ from measured trunk 
40 accelerometry under- or overestimated the $\mathrm{GRF}_{\text {param }}$ obtained from measured GRF, and

41 generally demonstrated larger within parameter variations.

42 Discussion. Despite the potential of obtaining a close fit between the MSD-model's upper mass

43 acceleration and the measured trunk accelerometry, the $\mathrm{ACC}_{\text {param }}$ estimated from this process

44 were inadequate to predict resultant GRF waveforms during slow to moderate speed running.

45 We therefore conclude that trunk-mounted accelerometry alone is inappropriate as input for

46 the MSD-model to predict meaningful GRF waveforms. Further investigations are needed to

47 continue to explore the feasibility of using body-worn micro sensor technology to drive simple

48 human body models that would allow practitioners and researchers to estimate and monitor

49 GRF waveforms in field settings. 


\section{Introduction}

52 Humans generate considerable forces against the ground during running to maintain an upright

53 posture. This comes at the cost of equal and opposite ground reaction forces (GRF) acting on

54 the body during every foot-ground contact (Cavanagh \& Lafortune, 1980). These GRF put the

55 body's soft tissues (e.g. bones, cartilage, muscles, tendons and ligaments) under biomechanical

56 stresses which over time are expected to lead to beneficial structural adaptations (Kibler,

57 Chandler \& Stracener, 1992; Dye, 2005). Inadequate recovery or repetitive GRF with excessive magnitudes can instead lead to negative adaptions and tissue damage (Kibler, Chandler \&

Stracener, 1992; Dye, 2005). The ability to monitor an athlete's GRF during running can

therefore help to better understand the relationship between the external forces experienced and soft-tissue adaptations (Vanrenterghem et al., 2017) ultimately helping to prevent musculoskeletal injury.

Accurate monitoring of GRF waveforms during running is currently restricted to laboratory environments where GRF waveforms are measured with force platforms built into the ground, or derived from whole-body kinematics (Bobbert, Schamhardt \& Nigg, 1991; Winter, 2005).

With recent developments of low-cost sensor based micro technology (Camomilla et al., 2018), accelerometry has become a popular tool to evaluate running mechanics outside laboratory environments in long and middle distance running (Tao et al., 2012) and professional team sports (Akenhead \& Nassis, 2016). Accelerometry also offers opportunities to estimate loading related GRF characteristics (e.g. Lafortune, 1991; Wundersitz et al., 2013; Neugebauer, Collins 
used as surrogate measure of peak GRF since the early 90s (Lafortune, 1991; Lafortune, Lake \& Hennig, 1995). However, recent studies found weak to moderate linear relationships between peak accelerations measured from body-worn accelerometry (trunk- and tibia-mounted accelerometers) and peak whole-body accelerations measured from force platforms during running (Wundersitz et al., 2013; Nedergaard et al., 2017a; Raper et al., 2018). Since body-worn accelerometers only measure segmental acceleration, the use of a single accelerometer has to date been inadequate to incorporate the complex multi-segmental accelerations that result in task-specific GRF patterns (Nedergaard et al., 2017a). Recent studies have indicated that from the combination of three or more body-worn inertial sensors and machine learning one can estimate GRF and knee joint moments with reasonable accuracy during running related locomotion (Johnson et al., 2018; Wouda et al., 2018), but the broader application of such approaches is constrained by the requirement of multiple sensors, machine learning tools, and large data sets. Therefore, if it were possible to estimate accurate GRF waveforms from a single body-worn sensor, it would provide practitioners and researchers with a useful tool to monitor the biomechanical load in field settings.

Since the overall motion of the human body has a spring-like behaviour during running, simple mass-spring models, consisting of a single mass and spring, have been widely used to estimate the vertical GRF in field settings (e.g. Alexander, 1984; Blickhan, 1989; McMahon \& Cheng, 1990). Moreover, such models have been used in combination with trunk-mounted accelerometry to estimate the required model parameters (Gaudino et al., 2013; Buchheit, Gray \& Morin, 2015). Unfortunately, the initial high-frequency impact peak typically observed in the 
95

96

97

GRF waveform during running, which is speculated to be linked with negative tissue adaptations and risk of injury (Nigg, Cole \& Bruggemann, 1995; Hreljac, Marshall \& Hume, 2000; Milner et al., 2006), cannot be estimated with this oversimplified model (Alexander, Bennett \& Ker, 1986; Bullimore \& Burn, 2007). A more complex mass-spring-damper model (MSD-model) better replicates the GRF waveforms for running at moderate speeds (3.83 $\mathrm{m} \cdot \mathrm{s}^{-1} \pm 5 \%$ ), including both impact and active peaks (Derrick, Caldwell \& Hamill, 2000). This model consists of a lower mass connected to a spring in parallel with a damper, representing the support leg during foot-ground contact, and an upper mass and spring representing the dynamics of the rest of the body. However, the ability to use trunk-mounted accelerometry to estimate the required model parameters for this model is yet unknown.

The aim of this study was to examine if the acceleration of the MSD-model's upper mass represents the acceleration of the trunk segment measured with trunk-mounted accelerometry during running. This hypothesis seems feasible, since the trunk segment represents half of the body mass (Dempster, 1955). If this provides a reasonable approximation, it might be feasible to estimate the required MSD-model parameters from trunk accelerometry to subsequently predict GRF from the MSD-model behaviour. Specifically, we therefore explored (1) the feasibility to estimate the MSD-model's eight natural model parameters from measured trunk accelerometry, and (2) whether these model parameters in fact predict reasonably accurate GRF waveforms during running at slow to moderate running speeds. 


\section{Materials \& Methods}

118 Subjects and protocol

119 Twenty healthy male athletes (age $22 \pm 4$ years, height $178 \pm 8 \mathrm{~cm}$, mass $76 \pm 11 \mathrm{~kg}$ ) who

120 engaged in running related sports activities on a weekly basis volunteered to participate in this

121 study. The institutional ethics committee at Liverpool John Moores University granted ethical

122 approval for this study (ethics approval number: 09/SPS/010) in accordance with the

123 Declaration of Helsinki, and written consent was obtained from all participants. After a 15

124 minute warm-up (including light jogging, dynamic stretching and individual dynamic tasks) and

125 an individual number of familiarisation trials, the participants were asked to run over a force

126 platform at different running speeds of $2,3,4$ and $5 \mathrm{~m} \cdot \mathrm{s}^{-1}( \pm 5 \%)$ in a randomised condition

127 order. Running speeds were measured with photocell timing gates (Brower Timing System,

128 Utah, USA) placed $2 \mathrm{~m}$ apart, with the last gate positioned $2 \mathrm{~m}$ before the centre of the force

129 platform as described in Vanrenterghem et al. (2012). The participants completed four trials of

130 each running speed, landing on the force platform with their dominant leg (defined as the self-

131 reported preferred kicking leg (van Melick et al., 2017)). Trials with unsuccessful foot contacts

132 on the force platform (double foot contact or when the foot was not placed within the force

133 platform) and/or when the desired approach speed was not achieved were repeated until a

134 valid trial was recorded.

Experimental measurements

137 Resultant ground reaction forces were measured (GRF) with a sampling frequency of $3000 \mathrm{~Hz}$

138 from a 0.9 x $0.6 \mathrm{~m}^{2}$ Kistler force platform (9287C, Kistler Instruments Ltd., Winterthur, 
139 Switzerland). Resultant trunk accelerations (TrunkAcc) were simultaneously collected at $100 \mathrm{~Hz}$

140 using a tri-axial accelerometer (KXP94, Kionex, Inc., Ithaca, NY, USA) with an output range of \pm

$14113 \mathrm{~g}$ embedded within a commercial GPS device (MinimaxX S4, Catapult Innovations, Scoresby,

142 Australia) with a total weight of 67 grams and $88 \times 50 \times 19 \mathrm{~mm}$ in dimension. The GPS device

143 was positioned on the dorsal part of the upper trunk between the scapulae within a small

144 pocket of a tight fitted elastic vest according to the manufacturer's recommendations (Boyd,

145 Ball \& Aughey, 2011). Different vest sizes were used to ensure the tightest fit for the individual

146 participants. TrunkAcc data (measured in the units g) was pre-processed with the

147 manufacturer's proprietary filter algorithms $(50 \mathrm{~Hz}$ low-pass filter, personal communication with

148 the manufacturer), and downloaded as 'raw accelerometer data' from the manufacturer's

149 software (Catapult Sprint, version 5.1.7, Melbourne, Australia) after each test session. Each

150 session also included a static measurement at beginning and end of the session to detect any

151 calibration drift over time, and none was detected. TrunkAcc and GRF were synchronised using

152 a combination of time and event synchronisation as described in Nedergaard et al. (2017a) and

153 exported to Matlab (version R2016a, The MathWorks, Inc., Natick, MA, USA) where a $4^{\text {th }}$ order

154 recursive Butterworth low-pass filter with a cut-off frequency of $20 \mathrm{~Hz}$ was applied to GRF and

155 TrunkAcc. GRF data was collected from a single stance phase per trial, where touch down and

156 take off were defined when the vertical GRF crossed a $20 \mathrm{~N}$ threshold.

158 Mass-spring-damper model

159 The complex multi-segmental dynamics of the human body during stance phase were modelled 160 as a passive MSD-model (Alexander, Bennett \& Ker, 1986; Derrick, Caldwell \& Hamill, 2000). 
161 This model consists of two masses (Fig. 1); a lower point mass $\left(m_{2}\right)$ on top of a linear spring $\left(k_{2}\right)$

162 in parallel with a damper $(c)$ representing the support leg; an upper point mass $\left(m_{1}\right)$

163 representing the dynamics of the rest of the body and another linear spring $\left(k_{1}\right)$ connecting the

164 two masses.

165

166 The one-dimensional motion of the MSD-model was described by the acceleration of the two

167 masses (Eqn. 5 and 6):

168

$169 \lambda=\frac{m_{1}}{m_{2}}$

$170 \quad \omega_{1}^{2}=\frac{k_{1}}{m_{1}}=\frac{(1+\lambda) k_{1}}{\lambda M}$

$171 \quad \omega_{2}^{2}=\frac{k_{2}}{m_{2}}=\frac{(1+\lambda) k_{2}}{M}$

$172 \zeta=\frac{c}{2 \sqrt{k_{2} m_{2}}}$

$173 \quad a_{1}=-\omega_{1}^{2}\left(p_{1}-p_{2}\right)+g$

$174 a_{2}=-\omega_{2}^{2} p_{2}+\omega_{1}^{2} \lambda\left(p_{1}-p_{2}\right)-2 \zeta \omega_{2}^{2} v_{2}+g$

$175 \quad G R F_{\text {model }}=\frac{M \omega_{2}}{1+\lambda}\left(\omega_{2} p_{2}+2 \zeta v_{2}\right)$

177 where $p_{1}, p_{2} v_{1}, v_{2}, a_{1}$, and $a_{2}$ are the initial positions, velocities and, accelerations of the two

178 masses $\left(m_{1}\right.$ and $\left.m_{2}\right)$, respectively; $\lambda$ is the mass ratio of the lower mass relative to the total

179 body mass (Eqn. 1); $\omega_{1}^{2}$ and $\omega_{2}^{2}$ are the natural frequencies of the springs (Eqn. 2 and Eqn. 3)

180 based on the linear spring constants $\left(k_{1}\right.$ and $\left.k_{2}\right)$ and the mass of the two masses $\left(m_{1}\right.$ and $\left.m_{2}\right) ; \zeta$

181 is the damping ratio of the damper (Eqn. 4); and $g$ is the acceleration from gravitational 
182

183 184 185

acceleration $\left(-9.81 \mathrm{~m} \cdot \mathrm{s}^{-1}\right)$. The resultant GRF acting on the MSD-model is calculated as in Eqn. 7, where $M$ is the sum of the two masses (i.e. total body mass):

\section{Model parameter estimation}

To estimate the eight MSD-model parameters $\left(p_{1}, p_{2} v_{1}, v_{2}, \omega_{1}^{2} \omega_{2}^{2}, \zeta, \lambda\right)$, we used gravity corrected TrunkAcc from the stance phase as a surrogate of the MSD-model's upper mass acceleration (Fig. 2A). For each trial, model parameters ( $\left.A C C_{\text {param }}\right)$ were optimised by fitting the MSD-model's upper mass acceleration $\left(a_{1}\right)$ to the TrunkAcc signal. A purpose-built gradient descent optimisation routine in Matlab was used, where the two second-order differential equations of the MSD-model's motion (Eqn. 5 and 6) were transformed to four first-order differential equations and solved numerically with a Runge Kutta $4^{\text {th }}$ order method. Root mean squared error (RMSE) between the TrunkAcc and $a_{1}$ waveforms were calculated for every iteration to determine the optimal model $\mathrm{ACC}_{\text {param }}$ combination that best fitted TrunkAcc for the individual trials. The $\mathrm{ACC}_{\text {param }}$ estimated from the TrunkAcc fitting were then used to predict the resultant GRF from Eqn. 7. Furthermore, to help understand differences in estimated model parameters and the predicted versus measured resultant GRF, we also estimated the eight model parameters ( $\mathrm{GRF}_{\text {param }}$ ) by fitting the MSD-model to the measured GRF (Fig. $2 \mathrm{~B}$ ), similar to the approach previously described by Derrick et al. (2000).

\section{Statistical analysis}

Measured and modelled GRF were normalised to the participants' mass. RMSE between the TrunkAcc and $a_{1}$, waveforms, and between the measured GRF and predicted GRF waveforms, 
204 were calculated to evaluate the accuracy of the TrunkAcc fitting and the predicted GRF,

205 respectively. RMSE median and interquartile range $\left(25^{\text {th }}\right.$ to $75^{\text {th }}$ percentile) were calculated to

206 determine the variation in the model's accuracy within and across running speeds. Similarly, the

207 median and interquartile range $\left(25^{\text {th }}\right.$ to $75^{\text {th }}$ percentile) of the $A C C_{\text {param }}$ and $\mathrm{GRF}_{\text {param }}$ were

208 calculated to explore the variation within and across running speeds. The median data

209 presented and discussed in the following is the median of all trials within the individual running

210 speeds ( $N=80$ trials) and the overall median across all running speeds ( $N=320$ trials).

211

212 Results

213 The first step was to estimate the required $\mathrm{ACC}_{\text {param }}$ that fit the MSD-model's upper mass

214 acceleration to the measured TrunkAcc signal. The MSD-model was able to fit the measured

215 TrunkAcc with good accuracy across running speeds, though $a_{1}$ systematically underestimated

216 the magnitude of the first peak observed in the accelerometry signal (Fig. 3A). The median

217 RMSE (interquartile range $25^{\text {th }}$ to $75^{\text {th }}$ percentile) of the TrunkAcc fitting increased from 0.16

$218(0.12 ; 0.22) \mathrm{g}$ at the slowest running speed to between $0.21(0.16 ; 0.26) \mathrm{g}$ and $0.22(0.16 ; 0.30)$

$219 \mathrm{~g}$ for three faster running speeds. Though similar median RMSE values were observed across

220 the three fastest running speeds, the interquartile range increased with increased running

221 speeds (Fig. 3A). Despite the good match between $a_{1}$ and TrunkAcc, poor GRF predictions were

222 observed across running speeds (Fig. 3B) and the median RMSE of the predicted GRF

223 systematically increased with running speeds, from $6.68(3.81 ; 15.30) \mathrm{N} \cdot \mathrm{kg}^{-1}$ at $2 \mathrm{~m} \cdot \mathrm{s}^{-1}$ to 12.77

$224(7.78 ; 27.22) \mathrm{N} \cdot \mathrm{kg}^{-1}$ at $5 \mathrm{~m} \cdot \mathrm{s}^{-1}$. 
226 Since the $\mathrm{ACC}_{\text {param }}$ resulted in poor GRF predictions, we next estimated the $\mathrm{GRF}_{\text {param }}$ by fitting

227 the MSD-model to the measured GRF waveforms (Fig. 2B) to determine if there was any

228 difference between the two sets of model parameters (Table 1 ) and to compare the upper mass

229 acceleration to the measured TrunkAcc. The MSD-model was able to replicate the measured

230 GRF with high accuracy when GRF param were estimated to directly fit the measured GRF (Fig.

231 4B). This was reflected in the low RMSE median and interquartile ranges observed across all

232 running speeds $\left(2 \mathrm{~m} \cdot \mathrm{s}^{-1}: 0.45(0.36 ; 0.60) ; 3 \mathrm{~m} \cdot \mathrm{s}^{-1}: 0.47(0.37 ; 0.61) ; 4 \mathrm{~m} \cdot \mathrm{s}^{-1}: 0.53(0.39 ; 0.66) ; 5\right.$

$233 \mathrm{~m} \cdot \mathrm{s}^{-1}: 0.59(0.46 ; 0.73)$; All Speeds: $\left.0.51(0.39 ; 0.64) \mathrm{N} \cdot \mathrm{kg}^{-1}\right)$. However, the MSD-model's upper

234 mass acceleration profiles then deviated considerably from the acceleration profiles measured

235 with trunk accelerometry (Fig. 4A). The $\mathrm{GRF}_{\text {param }}$ also differed considerably from the $\mathrm{ACC}_{\text {param }}$

236 (Fig. 4C and 4D). Namely, the $\mathrm{GRF}_{\text {param }}$ demonstrated smaller within parameter variation, which

237 was especially evident for $p_{2}$ and $v_{2}$. Also, lower $v_{1}$ (median difference $0.47 \mathrm{~m} \cdot \mathrm{s}^{-1}$ ) and higher $v_{2}$

238 (median difference $-1.73 \mathrm{~m} \cdot \mathrm{s}^{-1}$ ) values were observed across running speeds.

\section{Discussion}

241 This study illustrates that the MSD-model's upper mass acceleration could be fitted to the

242 measured trunk accelerometry with high accuracy, but the $\mathrm{ACC}_{\text {param }}$ estimated from this process

243 did not lead to accurate predictions of resultant GRF waveforms across a range of slow to

244 moderate running speeds. Further analysis of the MSD-model behaviour when fitting to the

245 measured resultant GRF revealed a considerable discrepancy in $\mathrm{GRF}_{\text {param }}$ compared to the

$246 \mathrm{ACC}_{\text {param }}$ when fitting the MSD-model to measured trunk accelerometry signals. These results 
247 demonstrate that our initial hypothesis that the MSD-model's upper mass acceleration

248 primarily represents the acceleration of the trunk was false.

Model parameter estimation

251 The eight model parameters are fundamental to calculating the resultant GRF acting on the

252 MSD-model, and though fitting TrunkAcc was successful, the $\mathrm{ACC}_{\text {param }}$ estimated from this

253 approach resulted in poor GRF predictions. Based on the equation of the upper mass

254 acceleration (Eqn. 5) and the $A_{C C}$ param estimated from TrunkAcc, it seems that the MSD-model

255 was able to fit the TrunkAcc by keeping the initial position of the upper mass $\left(p_{1}\right)$ and lower

256 mass $\left(p_{2}\right)$ low, and by keeping the spring stiffness of the upper spring $\left(\omega_{1}^{2}\right)$ low. Whereas $p_{1}$ has

257 minor influence on the predicted GRF, the velocity of the upper mass at initial contact $\left(v_{1}\right)$ is

258 indirectly influenced by changes in the initial upper mass position $\left(v_{1}=\dot{p_{1}}\right)$. Derrick et al.

259 (2000) found that decreased $v_{1}$ has a large impact on the duration of the stance phase and

260 therefore could have contributed to the overestimation of foot-ground contact (Fig. 3B).

261 Similarly, the MSD-model decreased the spring stiffness of the upper spring $\left(\omega_{1}^{2}\right)$ to better fit

262 the two acceleration peaks typically observed in the TrunkAcc data, which has previously been

263 shown to increase the duration of the stance phase (Derrick, Caldwell \& Hamill, 2000).

264 Furthermore, the MSD-model lowered the initial position of the lower mass $\left(p_{2}\right)$, which

265 previously has been shown to both increase the GRF at touch down and decrease the

266 magnitude of the impact peak (Derrick, Caldwell \& Hamill, 2000). We therefore believe that the

267 high GRF values observed in our GRF predictions at touch down (Fig. 3B) were primarily related

268 to the lower initial position of the lower mass $\left(p_{2}\right)$ required to fit the upper mass acceleration to 
269 the TrunkAcc. Finally, the MSD-model also kept the damping ratio $(\zeta)$ low to better fit the

270 magnitude of the two acceleration peaks in the TrunkAcc. Decreasing the damping ratio, has

271 however previously been shown to increase the oscillation in the model's GRF (Alexander,

272 Bennett \& Ker, 1986; Derrick, Caldwell \& Hamill, 2000), and may therefore explain why our GRF

273 predictions to a large extent include oscillating characteristics (Fig. 3B).

274

275 The comparison between the $\mathrm{ACC}_{\text {param }}$ estimated from the TrunkAcc and the $\mathrm{GRF}_{\text {param }}$ estimated

276 from measured GRF, clearly demonstrates that the model is unsuitable for predicting GRF from

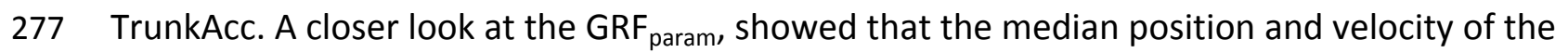

278 lower mass $\left(p_{2}\right.$ and $\left.v_{2}\right)$ was constant across running speeds and only varied marginally within

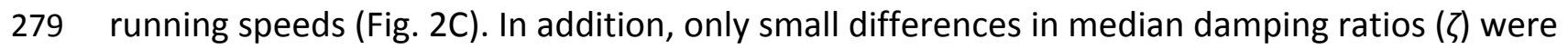

280 observed between running speeds in this study ( $\zeta$ between 0.31 and $0.39 \mathrm{au}$ ). It was in fact kept

281 constant $(\zeta=0.35 \mathrm{au})$ in the study by Derrick et al. (2000). Based on these observations we

282 explored the effect of keeping $p_{2}, v_{2}$, and $\zeta A C C_{\text {param }}$ constant for all trials (using the median

$283 \mathrm{GRF}_{\text {param }}$ across running speeds), and for the remaining five MSD-model parameters use the trial 284 specific $A C C_{\text {param }}$ to re-calculate the predicted GRF (Fig. S1). Whilst this decreased the variability 285 of the GRF prediction (RMSE interquartile range) both within and across running speeds, only 286 minor improvements were observed in the GRF prediction. This indicated that keeping selected $287 \mathrm{ACC}_{\text {param }}$ constant would not substantially improve the GRF prediction in future studies.

288 Furthermore, when selected $\mathrm{ACC}_{\text {param }}$ were kept constant, their original interaction was broken. 289 
291

292

293

294

295

296

297

298

299

300

301

302

303

304

305

306

307

308

309

310

311

312

MSD-model hypothesis

If the trunk accelerometry data accurately represents the model's upper mass acceleration one would at least expect that the $\mathrm{ACC}_{\text {param }}$ related to the motion and stiffness of the upper mass and spring $\left(p_{1}, v_{1}, \omega_{1}^{2}\right)$ would be close to the $\mathrm{GRF}_{\text {param }}$ estimated when fitting measured GRF.

This was however not the case, and therefore naturally raises the questions as to whether the upper mass acceleration is equivalent to the acceleration measured from trunk accelerometry during running. The trunk accelerometry driven MSD-model approach introduced in this study is based on the hypothesis that the model's upper mass primarily represents the mass and motion of the trunk segment (Alexander, Bennett \& Ker, 1986; Derrick, Caldwell \& Hamill, 2000). Our results suggest however that this is not the case, and that independent accelerations of other body segments (e.g. the swing leg and arms) significantly contribute to the MSD-model's upper mass accelerations. We therefore conclude that the primary model hypothesis for this study was false, and that trunk-mounted accelerometry alone is inappropriate as input for the MSD-model to predict meaningful GRF waveforms.

(1)

A high initial peak related to the attenuation of the shock impact from the foot's collision with the ground (Hamill, Derrick \& Holt, 1995; Derrick, 2004) dominated the TrunkAcc signals across running speeds. In contrast, a higher second peak related to the COM displacement during the stance phase dominated the upper mass acceleration when the MSD-model was fitted to measured GRF. This raised the technical question as to whether the poor GRF predictions observed from the measured accelerometer signal were partly a consequence of an artificially high frequency of that initial peak and whether the application of lower filter cut-off 
313 frequencies (cut-off frequencies of $20 \mathrm{~Hz}$ in the present study) would improve GRF predictions.

314 To explore this, trunk accelerometry data of 10 representative participants was low-pass

315 filtered with cut-off frequencies of 15, 10 and $5 \mathrm{~Hz}$ (Fig. S2). Whilst low cut-off frequencies

316 (especially 10 and $5 \mathrm{~Hz}$ ) to a large extent successfully removed the initial high-frequency peak in

317 the accelerometry signal, and the RMSE between TrunkAcc and upper mass acceleration

318 decreased, it only had a minor influence on the RMSE of the predicted GRF across running

319 speeds (Fig. S2). Therefore, accelerometry post-processing did not improve the GRF predictions

320 from TrunkAcc. This suggests that the trunk accelerometry signal in itself was not the main

321 reason for the poor GRF predictions, but rather an incorrect hypothesis that the MSD-model's

322 upper mass acceleration primarily represents the acceleration of the trunk segment.

\section{Replicating GRF from measured GRF}

325

326

327

328

329

330

331

332

333

334

Although TrunkAcc was unsuccessful in predicting GRF during running with a simple MSD-

model, the MSD-model could successfully replicate measured GRF during slow to moderate running speeds. In fact, the inclusion of all eight $\mathrm{GRF}_{\text {param }}$ in our optimisation routine, compared to only optimising the spring constants of the upper and lower spring $\left(k_{1}\right.$ and $\left.k_{2}\right)$ and the position of the lower mass $\left(p_{2}\right)$ (Derrick et al., 2000) allowed us to replicate the measured GRF with higher accuracy. These findings illustrate that despite the MSD-model simplicity it has the ability to replicate and potentially predict GRF for a range of running speeds. Since the MSDmodel parameters associated with the lower mass and spring are crucial to predict GRF (Eqn. 7), this may open opportunities to use segmental kinematics and/or accelerometry from lower extremities to estimate MSD-model parameters. This does however require that the lower limb 
335

336

337

338

339

340

341

342

343

344

345

346

347

348

349

350

351

352

353

354

355

356

accelerations measured from e.g. a tibia-mounted accelerometer are similar to the MSD-

model's lower mass acceleration required to accurately predict GRF, something which is not a given. Recent studies have for example shown promising results in predicting GRF during sprinting, in high level sprinters, when contact and flight time, in combination with kinematics from the ankle were used as input for a two-mass model (Udofa, Ryan \& Weyand, 2016; Clark, Ryan \& Weyand, 2017). Future studies are however still need to explore the use of body-worn micro sensor technology to drive simple human body models and predict GRF waveforms for a range running speeds.

\section{Model limitations}

A limitation with the MSD-model and the associated model parameters is that multiple parameter combinations exist when fitting the MSD-model to measured TrunkAcc or GRF waveforms. Whilst it could be of interest to further explore the physical meaning of the individual model parameters $\left(\mathrm{ACC}_{\text {param }}\right.$ or $\left.\mathrm{GRF}_{\text {param }}\right)$ and their interactions, or within and between subject parameters variations, this was not possible due to the existence of multiple model parameter solutions. Trunk-mounted accelerometry has a major benefit that it is already in use in many field contexts, but a limitation is that it may not very well represent the acceleration of the trunk segment. We have in previous work (Nedergaard et al. 2017b) shown that vertical trunk accelerations, measured from a high-end lab-based motion capture system, improved the upper mass acceleration fitting (median RMSE: 0.03g across all running speeds) and lowered the average median RMSE of the GRF predictions to $5.18 \mathrm{~N} \cdot \mathrm{kg}^{-1}$ (vertical GRF) across all running speeds, compared to $8.99 \mathrm{~N} \cdot \mathrm{kg}^{-1}$ in the current study. Importantly, the 
357 accuracy and reliability of the GRF predictions are considered poor in both cases, suggesting

358 that our hypothesis that the MSD-model's upper mass acceleration primarily represents the

359 trunk acceleration is most likely the weakest link. Secondly, the MSD-model is a one-

360 dimensional model, and therefore only allows the magnitude of the resultant GRF to be

361 estimated. We decided to predict the magnitude of the resultant GRF in our study, considering

362 that we wanted to estimate the overall external biomechanical loading on the body, however

363 we accept that others may prefer to predict the magnitude of the vertical GRF only. Ultimately,

364 we believe that it is important to recognise that the MSD-model approach omits any direction

365 specific load variations across running speeds, and that these may well be relevant in how the

366 musculoskeletal tissues are exposed to stresses. Finally, the MSD-model is a passive elastic

367 model and therefore does not account for additional energy generated by the body's "active"

368 structures (muscles). Whilst a more complex model could account for this (Zadpoor \&

369 Nikooyan, 2010; Nikooyan \& Zadpoor, 2011), it is questionable if this would allow for better

370 GRF predictions from TrunkAcc. The complexity of such model would probably also defeat the

371 overall purpose of using a simple model that is still applicable in field settings.

372

\section{Conclusions}

374 In this study, we demonstrated that the upper mass acceleration of a simple MSD-model can be

375 fitted to measured trunk accelerometry signals with high accuracy during running at various

376 speeds, but that the ensuing $\mathrm{ACC}_{\text {param }}$ do not deliver accurate predictions of GRF waveforms.

377 Despite the convenient hypothesis that the MSD-model's upper mass acceleration primarily

378 represents the acceleration of the trunk, our results showed that this hypothesis is violated too 
379 much to still predict meaningful GRF waveforms. Nevertheless, further studies should continue 380 to explore the use of data from wearable micro sensor technology to drive simple human body 381 models that could allow us to estimate GRF waveforms in field settings. This would allow 382 researchers and practitioners to better monitor the external biomechanical loads to which the 383 human body is exposed during running locomotion, ultimately supporting a general quest 384 towards field-based monitoring of tissue load-adaptation processes.

385

386 Acknowledgements

387 The authors would like to thank Ms Elena Eusterwiemann for her assistance with the data 388 collection. 
389

390

391

392

393

394

395

396

397

398

399

400

401

402

403

404

405

406

407

408

409

410

\section{References}

Akenhead R., Nassis GP. 2016. Training Load and Player Monitoring in High-Level Football: Current Practice and Perceptions. International Journal of Sports Physiology and Performance 11:587-593. DOI: 10.1123/ijspp.2015-0331.

Alexander RM. 1984. Elastic Energy Stores in Running Vertebrates. American Zoologist 24:8594. DOI: $10.1093 / \mathrm{icb} / 24.1 .85$.

Alexander RM., Bennett MB., Ker RF. 1986. Mechanical properties and function of the paw pads of some mammals. Journal of Zoology 209:405-419. DOI: 10.1111/j.14697998.1986.tb03601.x.

Blickhan R. 1989. The spring-mass model for running and hopping. Journal of Biomechanics 22:1217-1227. DOI: 10.1016/0021-9290(89)90224-8.

Bobbert MF., Schamhardt HC., Nigg BM. 1991. Calculation of vertical ground reaction force estimates during running from positional data. Journal of Biomechanics 24:1095-1105. DOI: 10.1016/0021-9290(91)90002-5.

Boyd LJ., Ball K., Aughey RJ. 2011. The Reliability of MinimaxX Accelerometers for Measuring Physical Activity in Australian Football. International Journal of Sports Physiology and Performance 6:311-321. DOI: 10.1123/ijspp.6.3.311.

Buchheit M., Gray AJ., Morin JB. 2015. Assessing Stride Variables and Vertical Stiffness with GPS-Embedded Accelerometers: Preliminary Insights for the Monitoring of Neuromuscular Fatigue on the Field. Journal of Sports Science and Medicine 14:698-701.

Bullimore SR., Burn JF. 2007. Ability of the planar spring-mass model to predict mechanical parameters in running humans. J Theor Biol 248:686-695. DOI: 10.1016/j.jtbi.2007.06.004. 
411 Camomilla V., Bergamini E., Fantozzi S., Vannozzi G. 2018. Trends Supporting the In-Field Use of

412 Wearable Inertial Sensors for Sport Performance Evaluation: A Systematic Review. Sensors $413 \quad$ 18:873. DOI: 10.3390/s18030873.

414 Cavanagh PR., Lafortune MA. 1980. Ground reaction forces in distance running. Journal of 415 Biomechanics 13:397-406. DOI: http://dx.doi.org/10.1016/0021-9290(80)90033-0.

416 Clark KP., Ryan LJ., Weyand PG. 2017. A general relationship links gait mechanics and running 417 ground reaction forces. The Journal of Experimental Biology 220:247-258. DOI: 10.1242/jeb.138057.

419

420

421

422

423

424

425

426

427

428

429

430

431

432

Dempster WT. 1955. Space requirements of the seated operator: geometrical, kinematic, and mechanical aspects of the body with special reference to the limbs. Wright-Patterson Air Force Base, Ohio: Wright Air Development Center.

Derrick TR. 2004. The effects of knee contact angle on impact forces and accelerations. Med Sci Sports Exerc 36:832-837. DOI: 10.1249/01.MSS.0000126779.65353.CB.

Derrick TR., Caldwell GE., Hamill J. 2000. Modeling the stiffness characteristics of the human body while running with various stride lengths. Journal of Applied Biomechanics 16:36-51. DOI: 10.1123/jab.16.1.36.

Dye SF. 2005. The pathophysiology of patellofemoral pain: a tissue homeostasis perspective. Clin Orthop Relat Res 436:100-110. DOI: 10.1097/01.blo.0000172303.74414.7d.

Gaudino P., Gaudino C., Alberti G., Minetti AE. 2013. Biomechanics and predicted energetics of sprinting on sand: hints for soccer training. J Sci Med Sport 16:271-275. DOI: 10.1016/j.jsams.2012.07.003.

Hamill J., Derrick TR., Holt KG. 1995. Shock attenuation and stride frequency during running. 
Human Movement Science 14:45-60. DOI: http://dx.doi.org/10.1016/01679457(95)00004-C.

435

436

437

438

439

440

441

442

443

444

445

446

447

448

449

450

451

452

453

454

Hreljac A., Marshall RN., Hume PA. 2000. Evaluation of lower extremity overuse injury potential in runners. Med Sci Sports Exerc 32:1635-1641.

Johnson WR., Mian A., Donnelly CJ., Lloyd D., Alderson J. 2018. Predicting athlete ground reaction forces and moments from motion capture. Med Biol Eng Comput. DOI: doi.org/10.1007/s11517-018-1802-7.

Kibler WB., Chandler TJ., Stracener ES. 1992. Musculoskeletal adaptations and injuries due to overtraining. Exerc Sport Sci Rev 20:99-126.

Lafortune MA. 1991. Three-dimensional acceleration of the tibia during walking and running. Journal of Biomechanics 24:877-886. DOI: 10.1016/0021-9290(91)90166-K.

Lafortune MA., Lake MJ., Hennig E. 1995. Transfer function between tibial acceleration and ground reaction force. Journal of Biomechanics 28:113-117. DOI: 10.1016/00219290(95)80014-X.

McMahon TA., Cheng GC. 1990. The mechanics of running: How does stiffness couple with speed? Journal of Biomechanics 23, Supple:65-78. DOI: http://dx.doi.org/10.1016/00219290(90)90042-2.

Milner CE., Ferber R., Pollard CD., Hamill J., Davis IS. 2006. Biomechanical factors associated with tibial stress fracture in female runners. Med Sci Sports Exerc 38:323-328. DOI: 10.1249/01.mss.0000183477.75808.92.

Nedergaard NJ., Robinson MA., Eusterwiemann E., Drust B., Lisboa PJ., Vanrenterghem J. 2017 a. The Relationship Between Whole-Body External Loading and Body-Worn Accelerometry 
457 Nedergaard, NJ., Robinson, MA., Drust, B., Lisboa, P., Vanrenterghem, J. 2017b. Predicting 458 ground reaction forces from trunk kinematics: A mass-spring-damper model approach. ISBS Proceedings Archive: Vol. 35: Iss. 1 , Article 24. Available at: https://commons.nmu.edu/isbs/vol35/iss1/24.

461

Neugerbauer, JM., Collins, KH., Hawkins, DA. 2014. Ground reaction force estimates from ActiGraph GT3X+ hip accelerations. PLoS ONE 9: e99023. DOI: 10.1371/journal.pone.0099023.

Nigg BM., Cole GK., Bruggemann GP. 1995. Impact forces during heel-toe running. Journal of Applied Biomechanics 11:407-432. DOI: 10.1123/jab.11.4.407.

Nikooyan AA., Zadpoor AA. 2011. An improved cost function for modeling of muscle activity during running. J Biomech 44:984-987. DOI: 10.1016/j.jbiomech.2010.11.032.

Raper DP., Witchalls J., Philips EJ., Knight E., Drew MK., Waddington G. 2018. Use of a tibial accelerometer to measure ground reaction force in running: A reliability and validity comparison with force plates. Journal of Science and Medicine in Sport 21:84-88. DOI: 10.1016/j.jsams.2017.06.010.

Tao W., Liu T., Zheng R., Feng H. 2012. Gait analysis using wearable sensors. Sensors 12:22552283. DOI: $10.3390 /$ s120202255.

Udofa AB., Ryan LJ., Weyand PG. 2016. Impact forces during running: Loaded questions, sensible outcomes. In: 2016 IEEE 13th International Conference on Wearable and Implantable Body Sensor Networks (BSN). 371-376. DOI: 10.1109/BSN.2016.7516290. 
477 Vanrenterghem J., Nedergaard NJ., Robinson MA., Drust B. 2017. Training Load Monitoring in

478 Team Sports: A Novel Framework Separating Physiological and Biomechanical Load-

479 Adaptation Pathways. Sports medicine (Auckland, N.Z.) 47:2135-2142. DOI:

480 $10.1007 / \mathrm{s} 40279-017-0714-2$.

481

482

483

484

485

486

487

488

489

490

491

492

493

494

495

496

497

498

Vanrenterghem J., Venables E., Pataky T., Robinson MA. 2012. The effect of running speed on knee mechanical loading in females during side cutting. Journal of Biomechanics 45:24442449. DOI: 10.1016/j.jbiomech.2012.06.029.

van Melick, N., Meddeler, BM., Hoogeboom, TJ., Nijhuis-van der Sanden, MWG., van Cingel, REH. 2017. How to determine leg dominance: The agreement between self-reported and observed performance in healthy adults. PLoS ONE 12: e0189876. DOI: 10.1371/journal.pone.0189876.

Winter DA. 2005. Biomechanics and motor control of human movement. Hoboken, N.J.: John Wiley \& Sons. DOI: 10.1002/9780470549148.

Wouda FJ., Giuberti M., Bellusci G., Maartens E., Reenalda J., van Beijnum B-JF., Veltink PH. 2018. Estimation of Vertical Ground Reaction Forces and Sagittal Knee Kinematics During Running Using Three Inertial Sensors. Frontiers in Physiology 9:1-14. DOI: 10.3389/fphys.2018.00218.

Wundersitz DW., Netto KJ., Aisbett B., Gastin PB. 2013. Validity of an upper-body-mounted accelerometer to measure peak vertical and resultant force during running and change-ofdirection tasks. Sports Biomech 12:403-412. DOI: 10.1080/14763141.2013.811284.

Zadpoor AA., Nikooyan AA. 2010. Modeling muscle activity to study the effects of footwear on the impact forces and vibrations of the human body during running. Journal of 
Biomechanics 43:186-193. DOI: 10.1016/j.jbiomech.2009.09.028. 
Figure 1

An illustration of the human body represented as a MSD-model.

The MSD-model consists of a lower mass spring damper element $\left(m_{2}, k_{2}, c\right)$ representing the support leg of the human body and an upper mass spring element $\left(m_{1}, k_{1}\right)$ representing the rest of the human body.
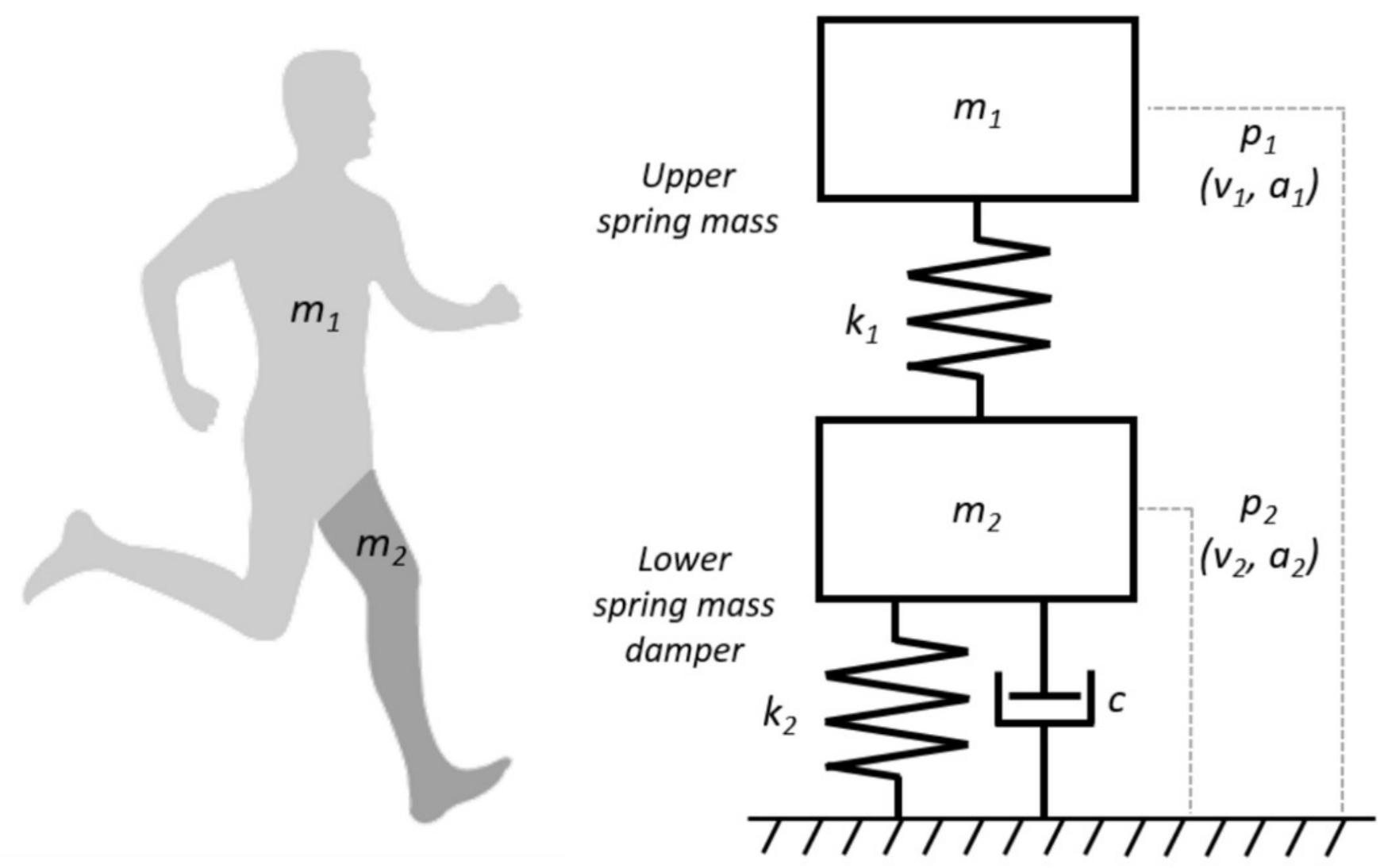


\section{Figure 2}

Estimating MSD-model parameters by fitting the MSD-model to measured trunk accelerometry and measured GRF.

Part A of Figure 2 illustrates the trunk driven MSD-model where measured trunk accelerometry (TrunkAcc) for the stance phase, is used to estimate the eight $\mathrm{ACC}_{\text {param }}$, based on the hypothesis that the MSD-model's upper mass acceleration (a1) primarily represents TrunkAcc, before GRF is calculated from the $\mathrm{ACC}_{\text {param }}$ that best fitted TrunkAcc. Part B of Figure 2 displays the traditional MSD- model approach, where the eight $\mathrm{GRF}_{\text {param }}$ are estimated by fitting the model's GRF to the measured GRF. 
A

Measure TrunkAcc

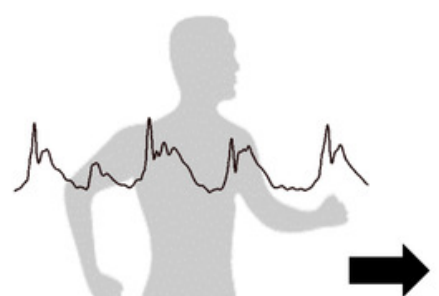

B

Measure GRF

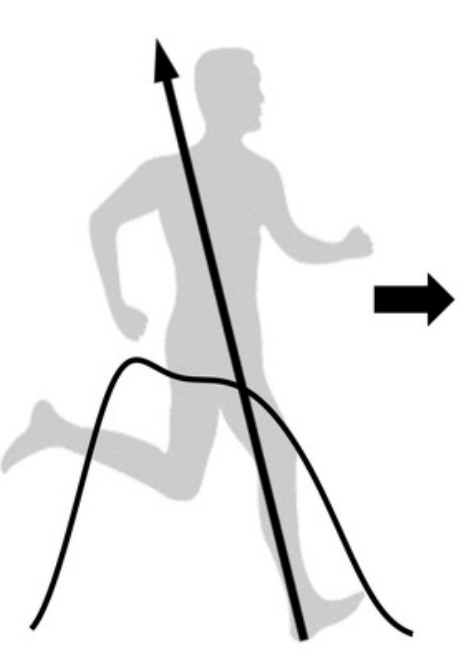

Fitting TrunkAcc To

Estimate $A C C_{p a r a m}$

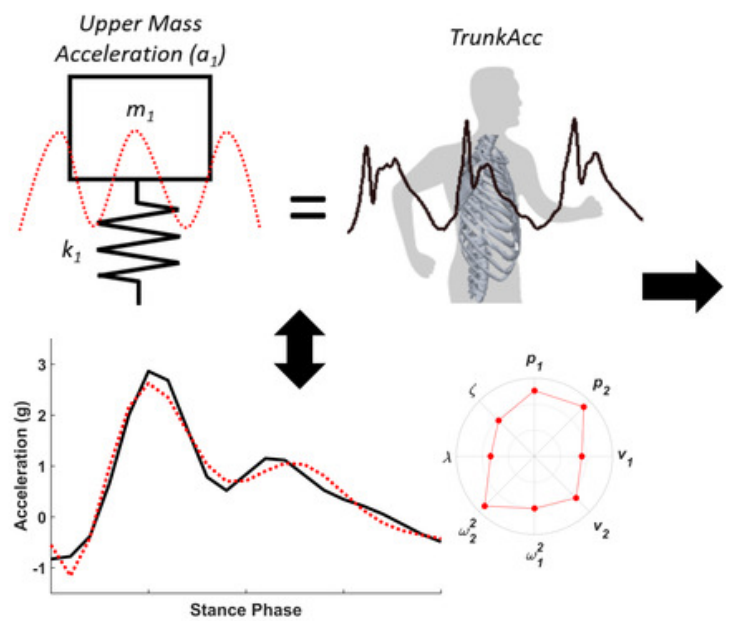

Fitting Measured GRF To

Estimate GRFparam
Predicting GRF From

Estimated ACCparam

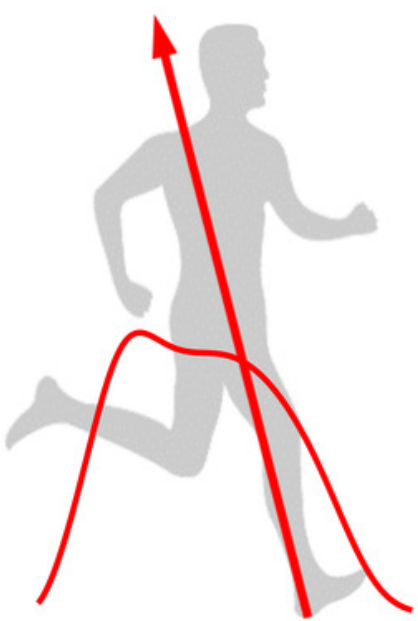

Replicating GRF From Estimated GRFparam
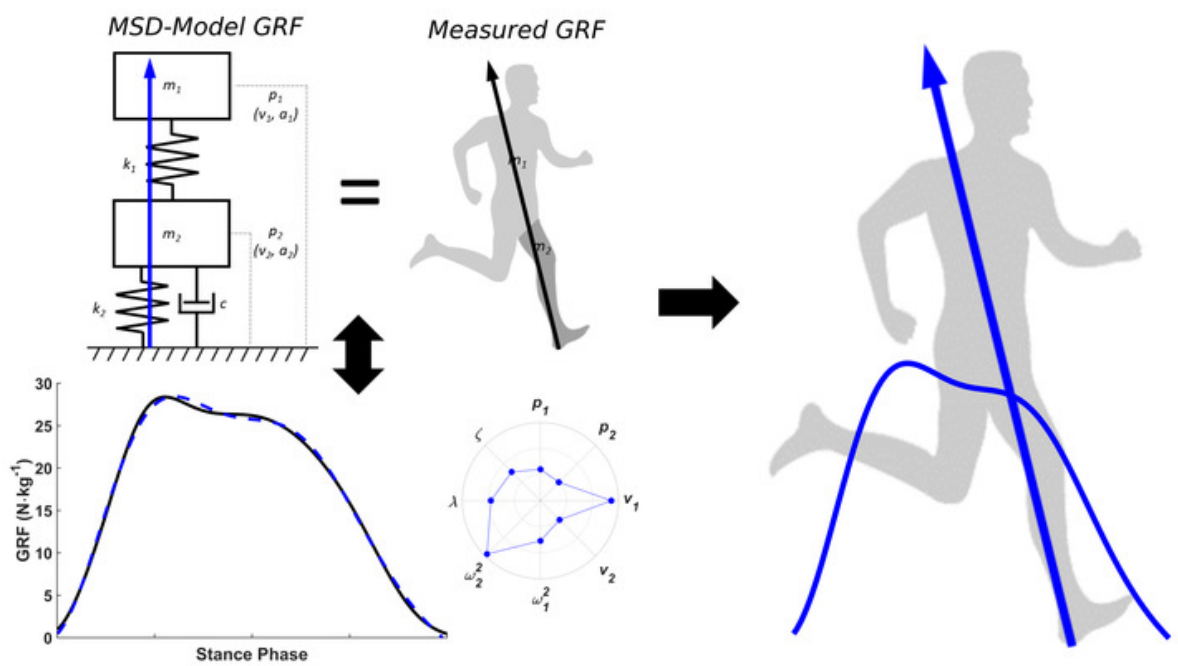


\section{Figure 3}

Representative examples of the trunk accelerometry fitting and GRF prediction, and the median RMSE across running speeds.

Representative examples of a single stride from multiple subjects. Part A of Figure 3 display the fitting the upper mass acceleration to the trunk accelerometry signal across running speeds, and part B of Figure 2 display the measured and predicted GRF for the same trials. The RMSE for the trunk accelerometry fitting and GRF predictions are displayed in brackets for the individual examples. The boxplots on the right side display the average RMSE median, and $25^{\text {th }}$ and $75^{\text {th }}$ interquartile range for the trunk accelerometry fitting and GRF prediction respectively within and across the individual running speeds. A total of 17 extreme outliers ( 3 $\mathrm{m} \cdot \mathrm{s}^{-1}: 3 ; 4 \mathrm{~m} \cdot \mathrm{s}^{-1}: 7 ; 5 \mathrm{~m} \cdot \mathrm{s}^{-1}: 7$ outliers) were removed through visual inspection from the boxplots in part B of Figure 3 to improve the visual interpretation.
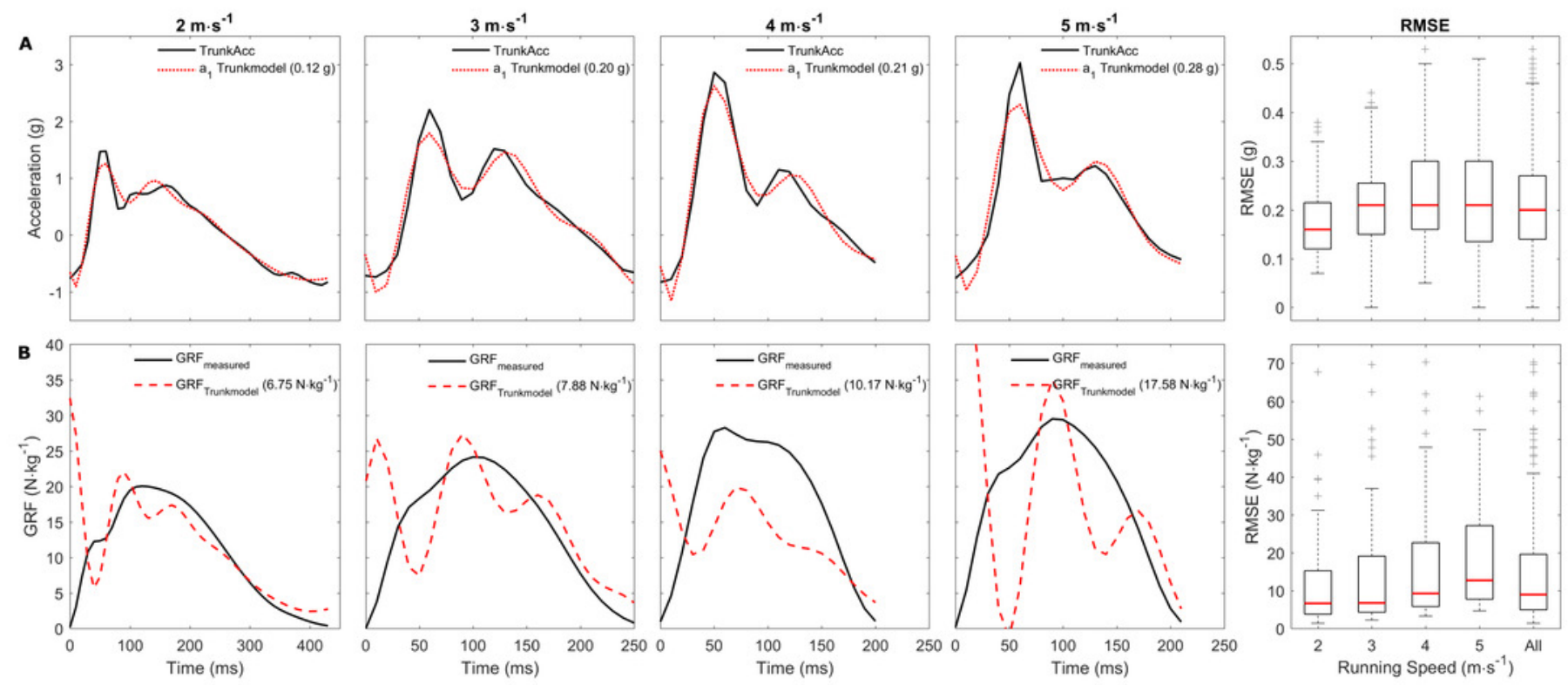


\section{Table $\mathbf{1}$ (on next page)}

Average median, $25^{\text {th }}$ and $75^{\text {th }}$ interquartile range for the $\mathrm{ACC}_{\text {param }}$ and $\mathrm{GRF}_{\text {param }}$ within and across the individual running speeds. 


\begin{tabular}{|c|c|c|c|c|c|}
\hline $\begin{array}{l}\text { Model } \\
\text { Parameters }\end{array}$ & $\begin{array}{c}2\left(\mathrm{~m} \cdot \mathrm{s}^{-1}\right) \\
\text { Median }\left(25^{\text {th }} ; 75^{\text {th }}\right)\end{array}$ & $\begin{array}{c}3\left(\mathrm{~m} \cdot \mathrm{s}^{-1}\right) \\
\text { Median }\left(25^{\text {th }} ; 75^{\text {th }}\right)\end{array}$ & $\begin{array}{c}4\left(\mathrm{~m} \cdot \mathrm{s}^{-1}\right) \\
\text { Median }\left(25^{\text {th }} ; 75^{\text {th }}\right)\end{array}$ & $\begin{array}{c}5\left(\mathrm{~m} \cdot \mathrm{s}^{-1}\right) \\
\text { Median }\left(25^{\text {th }} ; 75^{\text {th }}\right)\end{array}$ & $\begin{array}{c}\text { All } \\
\text { Median }\left(25^{\text {th }} ; 75^{\text {th }}\right)\end{array}$ \\
\hline \multicolumn{6}{|l|}{$p_{1}(m)$} \\
\hline ACCparam & $-0.02(-0.04 ;-0.01)$ & $-0.01(-0.04 ; 0.00)$ & $-0.02(-0.05 ; 0.00)$ & $-0.03(-0.06 ;-0.01)$ & $-0.02(-0.05 ;-0.01)$ \\
\hline GRFparam & $0.00(-0.01 ; 0.00)$ & $0.00(-0.01 ; 0.00)$ & $0.00(-0.02 ; 0.00)$ & $-0.01(-0.02 ;-0.01)$ & $-0.01(-0.02 ; 0.00)$ \\
\hline \multicolumn{6}{|l|}{$p_{2}(m)$} \\
\hline ACCparam & $-0.01(-0.02 ; 0.00)$ & $0.00(-0.02 ; 0.01)$ & $-0.01(-0.03 ; 0.00)$ & $-0.01(-0.04 ; 0.00)$ & $-0.01(-0.03 ; 0.00)$ \\
\hline GRFparam & $0.00(0.00 ; 0.01)$ & $0.00(0.00 ; 0.01)$ & $0.00(0.00 ; 0.00)$ & $0.00(0.00 ; 0.00)$ & $0.00(0.00 ; 0.00)$ \\
\hline \multicolumn{6}{|l|}{$v_{1}\left(m \cdot s^{-1}\right)$} \\
\hline ACCparam & $-0.58(-0.65 ;-0.50)$ & $-0.67(-0.82 ;-0.60)$ & $-0.71(-0.82 ;-0.60)$ & $-0.60(-0.69 ;-0.41)$ & $-0.64(-0.75 ;-0.54)$ \\
\hline GRFparam & $-0.91(-1.12 ;-0.72)$ & $-1.04(-1.26 ;-0.92)$ & $-1.24(-1.34 ;-1.11)$ & $-1.13(-1.27 ;-0.98)$ & $-1.11(-1.28 ;-0.92)$ \\
\hline \multicolumn{6}{|l|}{$v_{2}\left(m \cdot s^{-1}\right)$} \\
\hline ACCparam & $-1.98(-2.71 ;-1.37)$ & $-1.59(-2.91 ;-0.93)$ & $-1.78(-3.13 ;-1.20)$ & $-1.55(-2.39 ;-0.60)$ & $-1.75(-2.76 ;-1.08)$ \\
\hline GRFparam & $-0.02(-0.40 ; 0.00)$ & $-0.05(-0.28 ; 0.00)$ & $-0.01(-0.24 ; 0.00)$ & $0.00(-0.10 ; 0.00)$ & $-0.02(-0.25 ; 0.00)$ \\
\hline \multicolumn{6}{|c|}{$\omega_{1}^{2}\left(N \cdot m^{-1} \cdot \mathrm{kg}^{-1}\right)$} \\
\hline ACCparam & $334(233 ; 622)$ & $508(233 ; 966)$ & $477(315 ; 1193)$ & $512(331 ; 977)$ & $469(267 ; 958)$ \\
\hline GRFparam & $528(370 ; 721)$ & $577(357 ; 935)$ & $621(385 ; 959)$ & $687(495 ; 1025)$ & $604(411 ; 899)$ \\
\hline \multicolumn{6}{|c|}{$\omega_{2}^{2}\left(N \cdot m^{-1} \cdot k^{-1}\right)$} \\
\hline ACCparam & $2537(1167 ; 3895)$ & $2584(1152 ; 4174)$ & $2967(1628 ; 4688)$ & $3460(1517 ; 5094)$ & $2795(1320 ; 4362)$ \\
\hline GRFparam & $2421(1516 ; 3420)$ & $2966(2265 ; 4593)$ & $3574(2305 ; 5094)$ & $4006(3111 ; 6550)$ & $3253(2207 ; 4894)$ \\
\hline \multicolumn{6}{|l|}{$\lambda(\mathrm{au})$} \\
\hline ACCparam & $2.86(1.72 ; 4.60)$ & $2.61(1.46 ; 4.30)$ & $3.11(1.06 ; 4.27)$ & $2.25(0.97 ; 3.28)$ & $2.62(1.28 ; 4.13)$ \\
\hline GRFparam & $4.02(2.14 ; 6.62)$ & $5.19(2.82 ; 6.51)$ & $2.84(1.86 ; 5.90)$ & $2.74(1.82 ; 3.40)$ & $3.32(2.04 ; 5.84)$ \\
\hline \multicolumn{6}{|l|}{$\zeta(\mathrm{au})$} \\
\hline ACCparam & $0.23(0.18 ; 0.33)$ & $0.21(0.15 ; 0.32)$ & $0.20(0.15 ; 0.30)$ & $0.16(0.07 ; 0.32)$ & $0.20(0.15 ; 0.32)$ \\
\hline GRFparam & $0.38(0.29 ; 0.58)$ & $0.39(0.28 ; 0.51)$ & $0.37(0.28 ; 0.45)$ & $0.31(0.25 ; 0.40)$ & $0.36(0.27 ; 0.45)$ \\
\hline
\end{tabular}




\section{Figure 4}

Representative examples of the upper mass acceleration, GRF and median $\mathrm{ACC}_{\text {param }}$ and $\mathrm{GRF}_{\text {param }}$.

Representative examples of a single stride from multiple subjects. Part A of Figure 4 display the measured trunk accelerometry and the MSD-model's upper mass acceleration, and part B of Figure 4 display the measured, predicted and replicated GRF. The RMSE for the trunk accelerometry fitting and GRF predictions are displayed in the brackets for the individual examples. The inserted polar plots display the estimated model parameters (in unscaled values) from the two approached for the representative examples. Part C and D of Figure 4 display the average median, $25^{\text {th }}$ and $75^{\text {th }}$ interquartile range for the $\mathrm{ACC}_{\text {param }}$ and $\mathrm{GRF}_{\text {param }}$ within and across the individual running speeds. A total of 33 extreme outliers were removed from the $\mathrm{ACC}_{\text {param }}\left(p_{1}: 7 ; p_{2}: 8 ; v_{1}: 2 ; v_{2}: 13 ; \omega_{1}^{2}: 1 ; \lambda: 2\right.$ outliers) and 15 extreme outliers were removed from the $\mathrm{GRF}_{\text {param }}\left(\mathrm{v}_{1}: 6 ; \mathrm{v}_{2}: 1 ; \omega_{2}^{2}: 3 ; \lambda: 5, \zeta: 9\right.$ outliers) through visual inspection from the boxplots in part $C$ and $D$ of Figure 4 to improve the visual interpretation. 

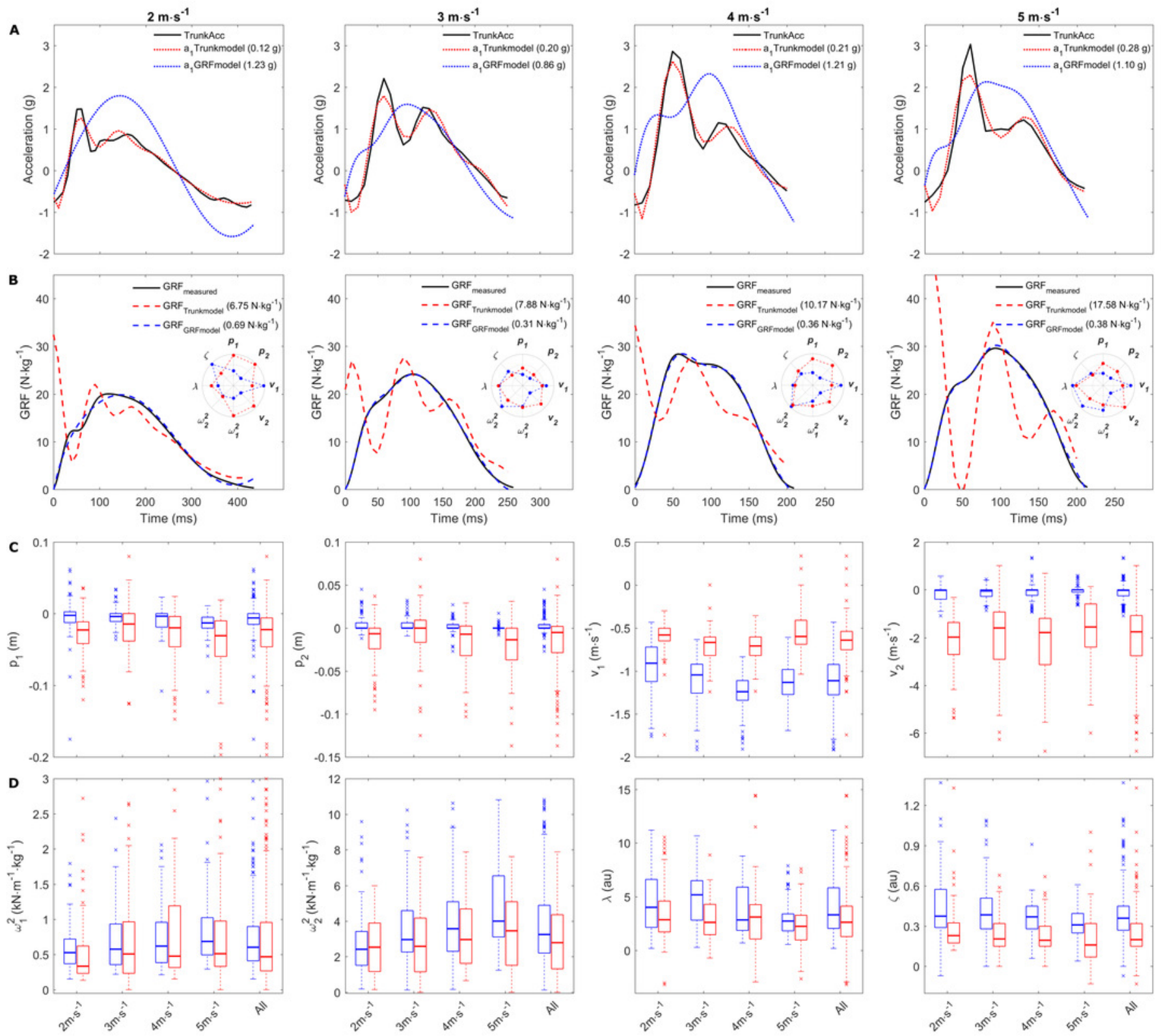